\title{
Lumbar Paraspinal Hemangiopericytoma, An Unusual Location: Surgery with Preoperative Embolization
}

\author{
Habib Abdoul Karim Ouiminga ${ }^{*}$, Aminath Kelani², Denléwendé Sylvain Zabsonre ${ }^{3}$, \\ Anatole Jean Innocent Ouedraogo', Desire Harouna Sankara4, Mengyou Li ${ }^{5}$, \\ Omar Salia ${ }^{1}$, Souleymane Ouattara ${ }^{6}$, Hongbo Wang ${ }^{7}$
}

\author{
${ }^{1}$ Department of Orthopedic, Traumatology and Neurosurgery, Tengandogo Teaching Hospital Center, Ouagadougou, Burkina Faso \\ ${ }^{2}$ Department of Neurosurgery, Niamey Teaching Hospital Center, Niamey, Niger \\ ${ }^{3}$ Department of Neurosurgery, Yalgado Ouedraogo Teaching Hospital Center, Ouagadougou, Burkina Faso \\ ${ }^{4}$ Department of Neuroradiology, Ouagadougou Medical Imaging Center, Ouagadougou, Burkina Faso \\ ${ }^{5}$ Department of Neurosurgery, Liaocheng People's Hospital, Liaocheng, China \\ ${ }^{6}$ Department of Pathology, CHU Tengandogo, Ouagadougou, Burkina Faso \\ ${ }^{7}$ Department of Orthopedic, Zaozhuang Municipal Hospital, Zaozhuang, China \\ Email: *ouimkar@gmail.com
}

How to cite this paper: Ouiminga, H.A.K., Kelani, A., Zabsonre, D.S., Ouedraogo, A.J.I., Sankara, D.H., Li, M.Y., Salia, O., Ouattara, S. and Wang, H.B. (2021) Lumbar Paraspinal Hemangiopericytoma, An Unusual Location: Surgery with Preoperative Embolization. World Journal of Neuroscience, 11, 108-115.

https://doi.org/10.4236/wins.2021.112009

Received: February 4, 2021

Accepted: April 24, 2021

Published: April 27, 2021

Copyright (c) 2021 by author(s) and Scientific Research Publishing Inc. This work is licensed under the Creative Commons Attribution International License (CC BY 4.0).

http://creativecommons.org/licenses/by/4.0/

\section{(c) (i) Open Access}

\begin{abstract}
Background: Lumbar paraspinal hemangiopericytoma is rare. The hemorrhagic nature of the tumor causes problems of hemostasis and makes the resection delicate. We report a rare case of lumbar paraspinal hemangiopericytoma managed with preoperative embolization. Clinical Case: It is about a woman of 31 years, admitted for a large left lumbar paraspinal mass that evolved for 6 years. The clinical exam showed an asymmetry of the left paraspinal muscle. The mass painless was extended from the lumbar region. The neurological exam was normal. MRI showed the left large paraspinal mass tissue. It was extended from lumbar vertebrae, L1 to L4, and measured $100 \times$ $50 \times 50 \mathrm{~mm}$. It was an iso-intense signal on T1-weighted with strong enhancement after gadolinium. It was hypervascular and supplied by left intercostal T12, L1, and L2 pedicles. The complete exclusion of the hyper-vascular left paraspinal tumor was obtained after selective embolization of the artery left L1 of the pedicle of the intercostal left L1 and trunks intercostal T12 and $\mathrm{L} 2$ left. The total resection of a mass encapsulated was performed. The operative outcome was good. The histology concluded to a hemangiopericytoma. No chemotherapy or radiotherapy was prescribed. After 5 years, the patient was asymptomatic. MRI control confirmed tumor resection with a residue at the level of the left intervertebral foramen L1 - L2. Conclusion: Lumbar paraspinal hemangiopericytoma is an extremely rare tumor. Selective preopera-
\end{abstract}


tive embolization is recommended before the resection of large tumors to reduce vascular supply. A follow-up extended for these patients is necessary, given the frequency of recurrences.

\section{Keywords}

Hemangiopericytoma, Embolization, Spine, Surgery

\section{Introduction}

Hemangiopericytoma is a rare mesenchymatous tumor from Zimmerman's pericytes [1] [2]. Although susceptible to growth in all tissues with a capillary vascularization, it accounts for $2 \%-3 \%$ of malignant soft tissue tumors of all locations [3]. Intracranial location is much more common than intraspinal location [4]. The mechanisms of hemangiopericytoma development remain poorly understood [4]. Treatment is based on surgical resection as wide as possible. The hemorrhagic nature of the tumor can cause major problems of hemostasis and make the resection delicate. To reduce tumor vascularization, preoperative embolization is sometimes performed to permit a complete resection [4] [5]. However, in clinical practice, the risk of local recurrence is high [4] [6]. This case illustrates a rare presentation of a lumbar paraspinal hemangiopericytoma managed with preoperative embolization.

\section{Clinical Case}

A 31-year-old woman had a left lumbar paraspinal mass that had been gradually evolving for 6 years. Clinical examination showed an asymmetry of the left paraspinal muscle compared to the right side. The mass was firm, painless, from left thoracolumbar seat to L1 to L4 with a skin coating was normal. The neurological examination was normal. Angio-MRI showed a large left lumbar paraspinal tissue mass. It measured $100 \times 50 \times 50 \mathrm{~mm}$. It was hypervascularized and fed by left T12, L1, and L2 intercostal pedicles. It appears in Iso-signal in T1-weighted and enhanced strongly after gadolinium (Figure 1). Its extension was extradural intra-foraminal of the left L2 root. A first-line mass biopsy had concluded that hemangiopericytoma. Complete exclusion of left paraspinal tumor hypervascularization was obtained after embolization of the left L1 main artery (Curaspon, embospheres 300 - $500 \mu$ ) of the left intercostal L1 pedicle (Glubran, Lipiodol) and left intercostal trunks T12 (Figure 2(A), Figure 2(B)) and L2 (Glubran, Lipiodol) (Figure 2(C), Figure 2(D)). After the posterior thoracolumbar median approach, a good hemostasis of the hemorrhagic tissues was performed. The dissection surrounds the mass was easy and progressive. The total resection of an encapsulated mass was carried out (Figure 3 ). The surgical outcome was simple. A thoracolumbar brace was prescribed for 6 weeks. Histology concluded that hemangiopericytoma. The cells were fusiform or oval with 
a rare cytoplasm. The mitotic index was minus 1 mitosis/10. There was a highly developed vascular component of the hemangiopericytic type, which was confirmed to immunohistochemistry (CD31). The patient received no chemotherapy or radiotherapy after surgery. The 3-month MRI confirms complete resection of the tumor as well as a lesion residue at the left L1 - L2 intervertebral foramen. The patient was asymptomatic after 5 years. The control MRI showed a stable lesion residue at the left L1 - L2 intervertebral foramen that enhances with contrast (Figure 4).
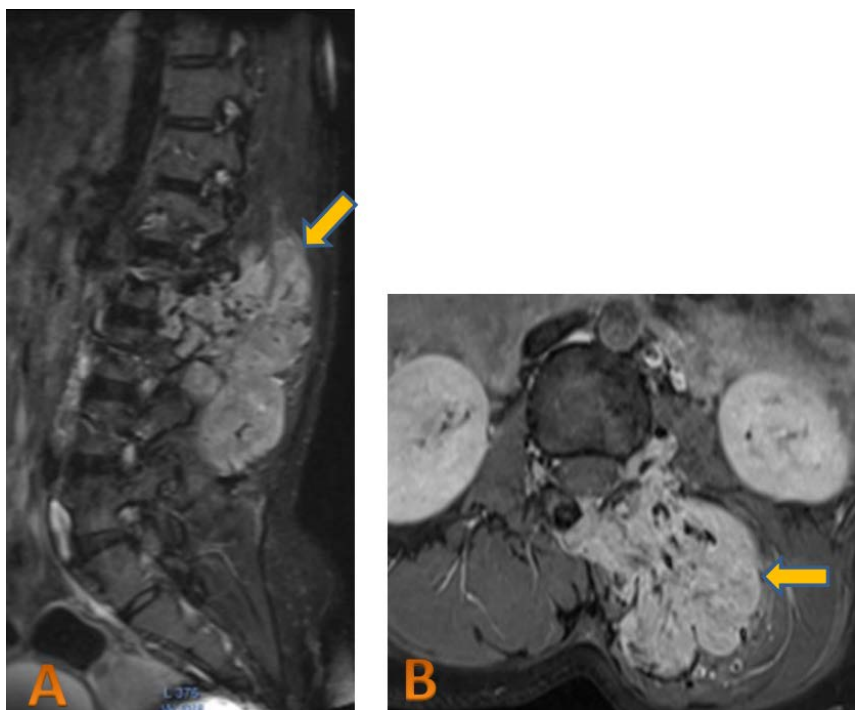

Figure 1. Large lumbar mass (Yellow arrow) extended to L1 to L4 (A), in T1-weighted and enhanced strongly after gadolinium with a foraminal extension (B).
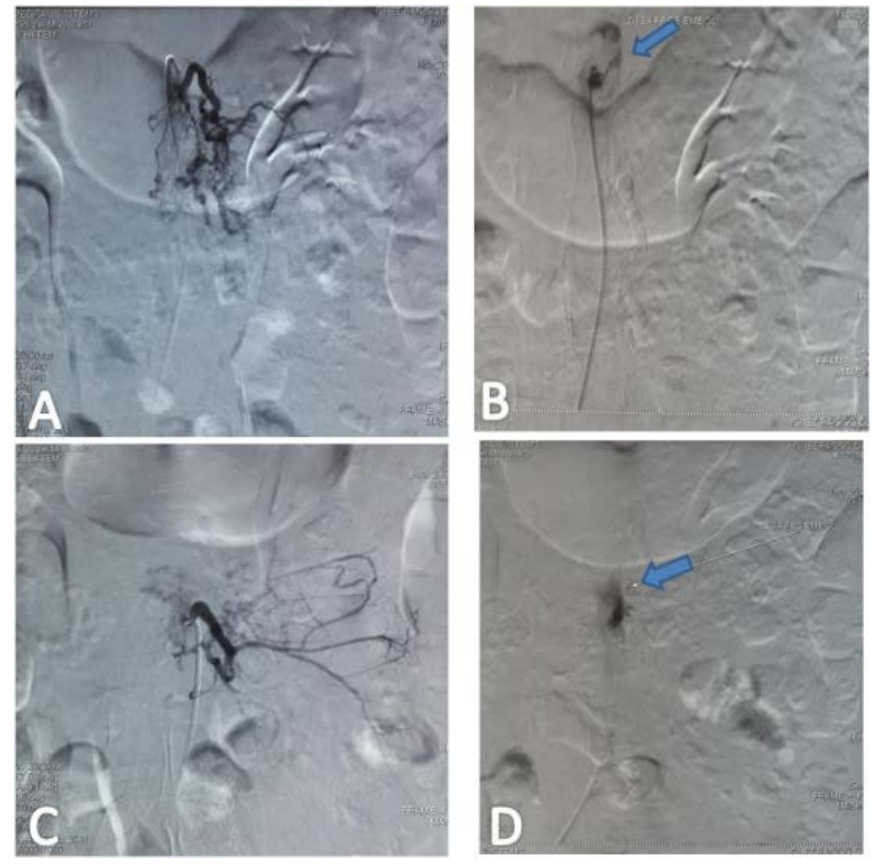

Figure 2. Preoperative embolization (A) T12 before embolization; (B) T12 after embolization (blue arrow); (C) L2 before embolization; (D) L2 after embolization (blue arrow). 


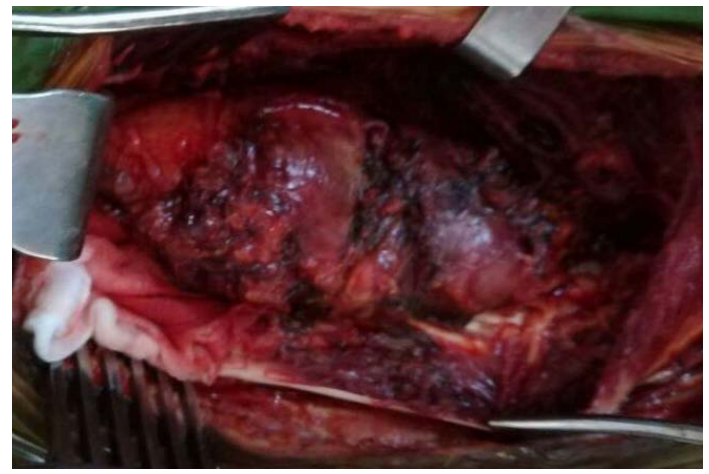

Figure 3. Per-operative image of the para-lumbar mass.
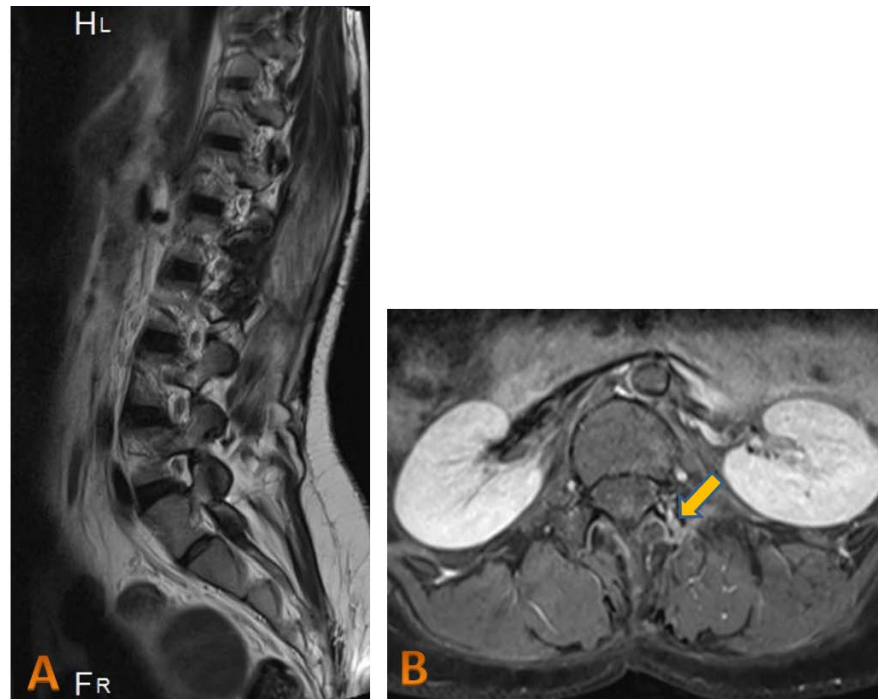

Figure 4. Post-operative follow-up MRI T2 -weighted confirms complete tumor resection (A) with a stable residue (Yellow arrow) at the left L1 - L2 intervertebral foramen (B).

\section{Discussion}

Hemangiopericytoma is a rare soft tissue tumor. It develops from Zimmerman pericytes which are extravascular cells found surround the capillaries or post-capillaries venules [6]. They belong to the group of solitary fibrous tumors [1] [2]. It particularly affects young adults. The average age of the patients reported by the authors is 31 years old [1] [7] [8]. Our patient is of this average age. The etiology of the disease is unknown. No existing clinical study has been able to link to specific causes. Hemangiopericytoma may occur anywhere in the body. The more frequent spinal locations described are cervical, thoracic, and rarer lumbar [3] [4] [9]. The particularity of the case illustrated in this writing differs from the lumbar spinal cases described in the literature by its extension. Only dural and extradural locations have been frequently described in the lumbar region [1], as we have observed in our case. She was extra-dural without neurological disorders with a muscular extension or intramuscular as described by the authors [9]. Rarely is it an extra-medullary intradural location [4] [8]. However, an intradural lesion could lead to root compression in the vertebral 
canal [4] [8] [9]. Symptoms of hemangiopericytoma lead to follow a slow and painless progression over several months or even years [8]. This slow-growing was also observed in our practice. This fact would explain the late discovery mode. The mass had been evolving for several years and initially painless. The symptoms mentioned in our practice were summed up in low back pain associated with the presence of a painless lumbar mass without a sensory-motor deficit. In other series, the authors report minor neurological disorders or stage neurological deficit [5] [8]. Angio-MRI, in our practice, provided details on the location of the tumor, its size, its relationship with adjacent structures. The greatest interest was the precision on the mass hypodensity characters in the T1-weighted and it's strong enhancing T1 gadolinium-weighted. It was used to evaluate the importance of its vascularization to guide the surgical procedure as described in the literature [5] [7] [10]. For the majority of authors, imaging is not very specific [3] [7]. It describes the characteristics of the tumor reminiscent of a Hemangiopericytoma. The similarity between their radiological traits and those of other tumors (Meningioma, neurofibroma) makes diagnosis difficult. Angiography is of particular interest because of the vascular origin of hemangiopericytoma [5] [7] [11]. It can sometimes be used, for preoperative embolization, or rarely postoperative, after surgical resection of the tumor with persistent arterial bleeding [5] [10]. In our practice, it had identified the branches of tumor supply and makes a preoperative embolization. The Complete exclusion of paraspinal tumor hypervascularization was a decisive step for our patient. This preoperative step for the removal of the tumor with minimal blood loss had been successful. It reduces blood loss per operative procedure and improves the surgeon's visibility and the quality of resection [10] [11]. Intraoperative spinal angiography and embolization can also be performed in cases of unexpected massive bleeding that is difficult to control during debulking [7]. This would facilitate the complete removal of the tumor in a narrow operating field. The embolization procedure can therefore be performed with a high success rate and allow surgery with maximum safety [10] [11] [12]. The treatment of hemangiopericytoma is primarily surgical [3] [6] [7] [10]. Other methods (embolization, irradiation, or chemotherapy) that can only be complementary, aimed at improving the local and general prognosis [6] [7]. The resection should be as complete as possible. Tumor resection was easy for our patient. It was carried out 24 hours after embolization of the main feeding arteries thus allowing control of blood loss. The debulking was carried out with good visibility, which allowed an almost complete ablation. A small fragment could not be extracted because attached to the root. Hemorrhagic tissues were accessible and hemostasis was easy. Preoperative embolization, used to reduce blood loss, increases the rate of total tumor resection [3] [5] [8] [11]. Generally, the diagnosis is confirmed by histology and immunohistochemistry [5] [7] [13] [14]. Histopathological aspects and CD31 markers were performed in our practice for hemangiopericytoma diagnosis. These imaging characteristics were similar to other solitary tumors. The im- 
agery would not have allowed them to be eliminated given the similarity of their radiological characters. Hemangiopericytoma should be differentiated from fibrous meningioma, fibrosarcoma, meningeal fibroblastoma, neurofibroma [7], [13]. The particularity of tumors is typically slow-growing, may be either benign or malignant. Vertebral hemangiopericytoma is described in the literature as a benign vascular tumor [1] [2]. The absence of necrosis and atypia and mitosis in our practice guarantees good biological behavior, WHO grade II [13]. However, for our patient, there was a tumor residue in MRI control. In the case of incomplete resection, postoperative radiotherapy is recommended [2] [8] [14] [15]. It should be proposed in cases of residual tumor or high-grade tumors [5] [8] [15]. The option of annual monitoring (control MRI) was proposed for our patient. The radiotherapy and the chemotherapy had not been prescribed for our patient. All the more so the histological traits of the lesion showed no signs of malignancy. The residue could be post-operative fibrosis that enhances with contrast. No significant improvement was reported in the clinical outcomes after radiation therapy [14]. Chemotherapy used as a treatment, adjuvant did not show its evidence in published series [3] [4] [6]. The management of these tumor residues is a therapeutic challenge as the tumor may have an unpredictable progression to an anaplastic hemangiopericytoma, WHO Grades III [1] [2] [9] [13]. The frequency of recurrence varies from $48 \%$ to $88 \%$ depending on the series [1] [3] [4] [12]. The prognosis depends on the site, the dimensions, and the histological grade of the tumor [9]. A small, less than $5 \mathrm{~cm}$, the intradural and dural tumor has a better prognosis [3] [4] [9]. For our patient, the size of the tumor was much larger. The histological characters had a benign profile. Surgical resection is the fundamental treatment and was associated with strict monitoring in the long term as recommended by the authors [2] [6] [8] [9]. The prognosis is good after complete resection and functional recovery is expected in the majority of patients [8] [12] [14] [15]. In some cases, the evolution was favorable as we described for our patient. Indeed, some publications report healings, followed for more than 10 years, after local treatment [3] [8]. In our case, 5 years after tumor resection, the residue is stable. No recurrences were observed. The follow-up was continued. Total resection initial surgery is associated with a lower risk of recurrence.

\section{Conclusion}

Lumbar Paraspinal hemangiopericytoma is an extremely rare tumor. Surgical challenges are mainly related to the hypervascularization of the tumor. Preoperative embolization is an important aid for the surgery, because of the excessive bleeding associated with these big size tumors. Total tumor resection is associated with good prognosis. Prolonged follow-up is necessary, given the frequency of recurrences.

\section{Acknowledgements}

Consent has been obtained from the patient for publication of this manuscript 
and all that accompany the images.

\section{Conflicts of Interest}

The authors declare no conflicts of interest regarding the publication of this paper.

\section{References}

[1] Ramdasi, R.V., Nadkarni, T.D. and Goel, N.A. (2014) Hemangiopericytoma of the Cervical Spine. Journal of Craniovertebral Junction and Spine, 5, 95-98.

https://doi.org/10.4103/0974-8237.139209

[2] Ventrapati, P., Pathy, S., Gandhi, A.K. and Kashyap, S. (2017) Anaplasic Hemangiopericytoma of Eyelid: An Unusual Location. Journal of Cancer Research and Therapeutics, 13, 145-147. https://doi.org/10.4103/0973-1482.206240

[3] Liu, H.G., Yang, A.C., Chen, N., Yang, J., Qiu, X.G. and Zhang, J.G. (2013) Hemangiopericytomas in the Spine: Clinical Features, Classification, Treatment, and Long-Term Follow-Up in 26 Patients. Neurosurgery, 72, 16-24. https://doi.org/10.1227/NEU.0b013e3182752f50

[4] Shirzadi, A., Drazin, D., Gates, M., Shirzadi, N., Banykh, S., Fan, X., et al. (2013) Surgical Management of Primary Spinal Hemangiopericytomas: An Institutional Case Series and Review of the Literature. European Spine Journal, 22, S450-S459. https://doi.org/10.1007/s00586-012-2626-Z

[5] Cole, C.D. and Schmidt, M.H. (2009) Hemangiopericytomas of the Spine: Case Report and Review of the Literature. Rare Tumors, 1, e43. https://doi.org/10.4081/rt.2009.e43

[6] Wang, K., Mei, F., Wu, S. and Tan, Z. (2020) Hemangiopericytoma: Incidence, Treatment, and Prognosis Analysis Based on SEER Database. BioMed Research International, 2020, Article ID: 2468320. https://doi.org/10.1155/2020/2468320

[7] Lee, C.-H., Kim, K.-J., Jahng, T.-A. and Kim, H.-J. (2013) Spinal Hemangiopericytoma Which Needed Intraoperative Embolization Due to Unexpected Bleeding. Journal of Korean Neurosurgical Society, 54, 253-256. https://doi.org/10.3340/jkns.2013.54.3.253

[8] Singh, P., Singla, R., Chandra, P.S., Kale, S.S. and Mahapatra, A.K. (2017) Rare-34. An Institutional Review of Nine Cases of Spinal Hemangiopericytoma. Neuro-Oncology, 19, vi217. https://doi.org/10.1093/neuonc/nox168.876

[9] Boyett, D., Kinslow, C.J., Bruce, S.S., Sonabend, A.M., Rae, A.I., McKhann, G.M., et al. (2019) Spinal Location Is Prognostic of Survival for Solitary-Fibrous Tumor/Hemangiopericytoma of the Central Nervous System. Journal of Neuro-Oncology, 143, 457-464. https://doi.org/10.1007/s11060-019-03177-0

[10] Nair, S., Gobin, Y.P., Leng, L.Z., Marcus, J.D., Bilsky, M., Laufer, I., et al. (2013) Preoperative Embolization of Hypervascular Thoracic, Lumbar, and Sacral Spinal Column Tumors: Technique and Outcomes from a Single Center. Interventional Neuroradiology, 19, 377-385. https://doi.org/10.1177/159101991301900317

[11] El Hindy, N., Ringelstein, A., Forsting, M., Sure, U. and Muelle, O. (2013) Spinal Metastasis from Malignant Meningeal Intracranial Hemangiopericytoma: One-Staged Percutaneous Onyx ${ }^{\mathrm{Tm}}$ Embolization and Resection-A Technical Innovation. World Journal of Surgical Oncology, 11, 152. https://doi.org/10.1186/1477-7819-11-152

[12] Champeaux, C., Khan, A.A., Wilson, E., Thorne, L. and Dunn, L. (2018) Solitary 
Fibrous Tumors and Haemangiopericytoma of the Meninges. A Retrospective Study for Outcome and Prognostic Factor Assessment. Neurochirurgie, 64, 37-43. https://doi.org/10.1016/j.neuchi.2017.10.004

[13] Louis, D.N., Perry, A., Reifenberger, G., Von Deimling, A., Figarella Branger, D., Cavenee, W.K., et al. (2016) The 2016 World Health Organization Classification of Tumors of the Central Nervous System: A Summary. Acta Neuropathologica, 131, 803-820. https://doi.org/10.1007/s00401-016-1545-1

[14] Chew, L.S., Han, X.J., Tan, K.K. and Bundele, M.M. (2017) Hemangiopericytoma of the Thoracic Spine: A Case Report. Journal of Surgical Case Reports, 7, 1-4. https://doi.org/10.1093/jscr/rjx121

[15] Singla, R., Singh, P., Khanna, G., Suri, V., Agrawal, P., Chandra, P.S., et al. (2020) An Institutional Review of 10 Cases of Spinal Hemangiopericytoma/Solitary Fibrous Tumor. Neurology India, 68, 448. https://doi.org/10.4103/0028-3886.284374 